\title{
IV
}

\section{Stellar activity}

- Surface mapping of active stars

- Stellar cycles, starspots and plages

- Stellar magnetic field measurements

- Chromospheric and coronal activity as a dynamo indicator

- Evolution of magnetic activity, activity in pre-MS-stars, subgiants and giants 


\title{
The Differential Rotation and Evolution of Spots on UX Arietis from a Sequence of Doppler Images
}

\author{
Steven S. Vogt and Artie P. Hatzes \\ University of California Observatories/ Lick Observatory, U. of California, \\ Santa Cruz, CA 95064
}

\begin{abstract}
We present a sequence of three Doppler images of the spotted RS CVn star UX Arietis obtained over a 5-month interval from August 1986 through January 1987. The spot distribution was quite complex and consisted of a large stable polar spot, a spot near the equator, and several other spots at intermediate positive and negative latitudes. The time intervals between successive images were small enough that we were able to reliably track the evolution of the spot distribution, measuring accurate longitudes, latitudes, and areas of the major spots, as well as their drift rates in longitude and latitude. The longitudinal drifts of spot features at equatorial, intermediate, and high latitudes yielded an accurate measurement of differential rotation. We find that the spotted primary of UX Arietis is indeed rotating differentially and in the sense opposite to that of the Sun, i.e. the poles rotate faster than the equator. The equator is synchronized to the orbital angular velocity, and the angular velocity increases towards either pole. The angular velocity distribution can be expressed as $\Omega\left({ }^{\circ} /\right.$ day $)=-55.91+1.09( \pm 0.09) \sin ^{2} \phi$, where $\phi$ is the latitude. The amount of differential rotation, parameterized as the ratio of the difference between the equatorial and polar angular velocities to the equatorial angular velocity, is then $\alpha=-0.020( \pm 0.002)$, as compared to a value of $\alpha=+0.2$ for the Sun.
\end{abstract}

\section{Introduction}

For the past 9 years, we have been obtaining Doppler images of a set of relatively bright spotted RS CVn stars. Our aim is to track the emergence and evolution of spots on these stars from one year to the next so as to obtain information on the systematics, patterns, and periodicities in spot behavior as well as to provide a direct measurement of differential rotation or latitudinal shear. Such information will provide insight into the underlying dynamo processes and large scale global convective and circulation patterns in much the same way as long term monitoring of sunspots on the Sun has revealed the 11-year and 22-year sunspot cycle and 'butterfly' diagram. These systematics are important observational anchor points 
for theories of the solar dynamo, and, through Doppler imagery of starspots, we hope to provide similar observational anchor points for theories of the extremely powerful dynamos of the RS CVn stars.

The technique we use is called Doppler imaging (Vogt et al., 1987; hereafter Paper I). Doppler imaging exploits the correspondence between wavelength position across a rotationally Doppler-broadened spectral line and spatial position across the stellar disk to derive a 2 -d image of the star. Cool spots on a rotating star produce distortions in the star's spectral line profiles. If the line profiles are dominated by rotational broadening, a high degree of correlation exists between the position of a given distortion in a line and the longitude on the star of the feature which produced it. A high resolution spectrum of the line is thus, to first order, a 1-d image of the star in longitude, but completely blurred in latitude. As the star is observed from other aspects (other rotation phases) additional 1$\mathrm{d}$ images are obtained. Then, if the inclination, rotation period, and other basic physical parameters of the star are known, the set of 1-d images can be combined into a 2-d image of the star.

We use Doppler imagery since the other available method of obtaining information on spot sizes and locations, modeling integrated flux light curves, is highly nonunique. There just isn't enough information in integrated flux light curves to be able to determine accurate longitudes and latitudes of multiple spots on a star, nor to extract any detail about the structure and shapes present in a complex spot distribution.

UX Arietis is one of our primary Doppler imaging candidates. It is a bright, noneclipsing RS CVn spectroscopic triple system. It consists of a spotted K0 IV primary and a G5 V secondary in a 6.44 day orbit. In the course of our Doppler imaging studies, we noticed a faint third component in the spectra which remains stationary near the $\gamma$ velocity of the system and which is apparently from a star in a long-period orbit about the binary.

In this contribution, we present a sequence of three Doppler images of the spotted K0 IV primary of the UX Arietis system which we obtained in late 1986 and early 1987, and from which we are able to derive an accurate measurement of differential rotation. For this symposium, we will present only a brief overview of our findings.

\section{Observations}

Data were acquired using the coude focus of the Shane 3-meter telescope at Lick Observatory and a TI $800 \times 8003$-phase CCD. The observations were centered on $6435 \AA$ and covered the wavelength region from about $6425 \AA$ to $6453 \AA$. The spectral resolution was about $0.13 \AA$. A modified Bowen-Walraven image slicer was used to reformat the light from a 3-arcsecond diameter hole to a 0.67 -arcsecond slit. Slicing not only minimized slit losses due to seeing but it also spread the light (perpendicular to dispersion) over a larger area of the CCD. This accomplished two things important for acquisition of the high signal-to-noise required for Doppler 


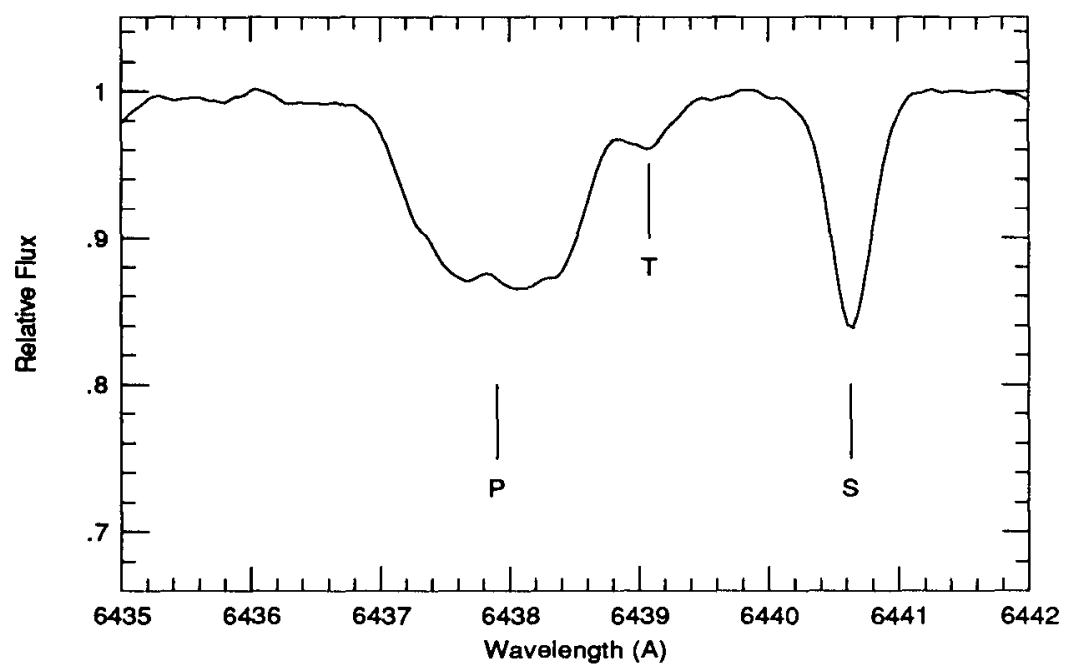

Fig. 1. Spectral region around Ca I $6439 \AA$ in UX Arietis showing the three stellar components.

imaging: it allowed for a higher total photon count without saturation of individual CCD pixels and it removed any additional pixel-to-pixel variations that may have been present after performing standard flatfielding.

It is important not only to have data with low noise, but also to remove the contribution of atmospheric features and stellar companions which may cause systematic errors. This is somewhat complicated for UX Arietis which is actually a triple system. Figure 1 shows an observation of Ca I $6439 \AA$ when all three components are visible. Before deriving the Doppler image care was taken to remove both secondary and tertiary components from the observed spectral line profiles.

The Ca I $6439 \AA$ line was used for modeling the spot distribution on the stellar surface. The data set was divided into three subsets. The 'August' set consisted of 4 observations whose median date was 18 August 1986. The time span of the observations was 4 days ( 0.6 rotations). The resulting Doppler image thus represents a 'snapshot' of the star in that the spot distribution would have changed little during the course of the observations. However, the phase coverage is incomplete. The observed $\mathrm{Ca}$ I profiles as a function of phase are shown as crosses in Figure 2. The fit to the observed spectral line profiles from the derived spot distribution is shown as a solid line.

The 'November' data set consisted of 5 phases (partial phase coverage) with a median date of 18 November 1986. Virtually all the observations were taken in December with the exception of one taken at phase 0.207 in October. This time span is long enough that if the spots had moved significantly during this time then the Doppler image would show some phase smearing, but only around phase 0.2 . The observed Ca I spectral line profiles as well as linefits are shown in Figure 3. 

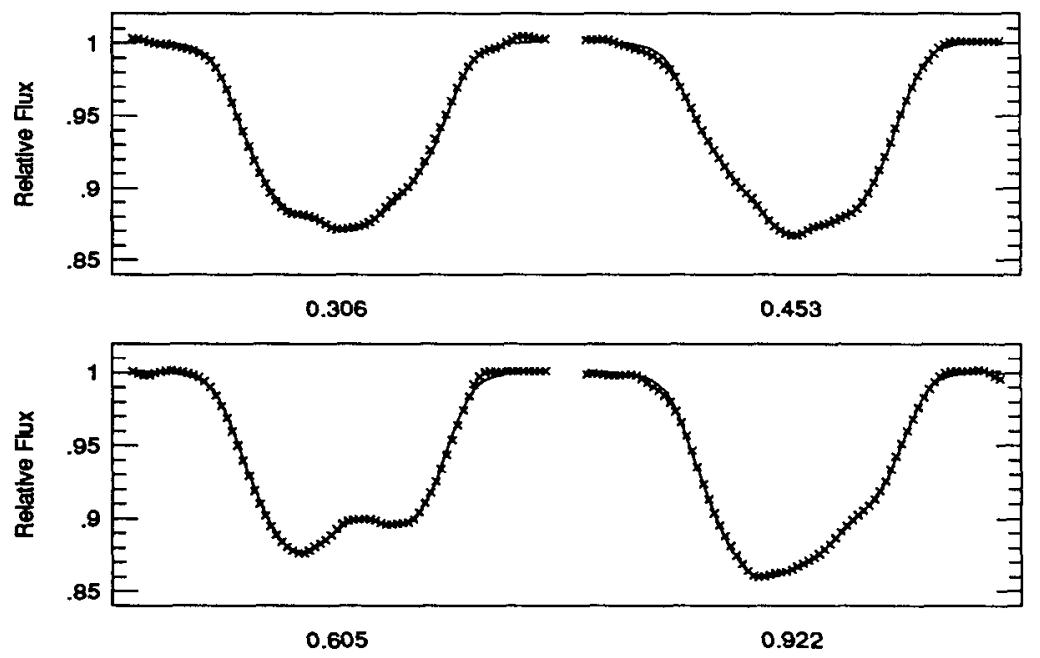

Fig. 2. The observed spectral line profiles (crosses) for Ca I as a function of phase for the August data set. The solid line represents the fits to the profiles from the derived spot distribution.

The 'January' data set represents our best data set for the observing season. The observations were all taken within an 8 day time span (1.3 rotations) centered on 16 January 1987. The phase smearing due to spot migration is thus negligible. This is the only data set for which we have complete phase coverage for the star. Again the observed line profiles are shown as crosses and the linefits as a solid line in Figure 4.

\section{Modeling the spot distribution on UX Arietis}

\subsection{Stellar parameters}

The adopted parameters used for modeling the spot distribution on UX Arietis are shown in Table 1. The parameters for the spotted primary are appropriate for a K0IV star and include the projected rotational velocity $v \sin i$, the inclination $i$, the temperature of the photosphere $\mathrm{T}_{p h o t}$, the logarithm of the surface gravity $\log g$, the radial-tangential macroturbulent velocity $\xi_{r t}$, and the coefficient for the standard limb darkening law $\epsilon$. The parameters for the secondary component are also listed and are appropriate for a G5V star. These were used to create a synthetic profile for the secondary which was subtracted from those observations where the primary and secondary were blended. For the third component we found that its profile could be adequately fit with a scaled version of the secondary profile. This component appeared to be stationary at $6.6 \mathrm{~km} \mathrm{~s}^{-1}$ blueward of the $\gamma$ velocity of the system.

Specific intensity profiles for the Ca I line were generated using model atmospheres from Bell et al. (1976) and Kurucz's WIDTH5 program. Thirty limb angles 

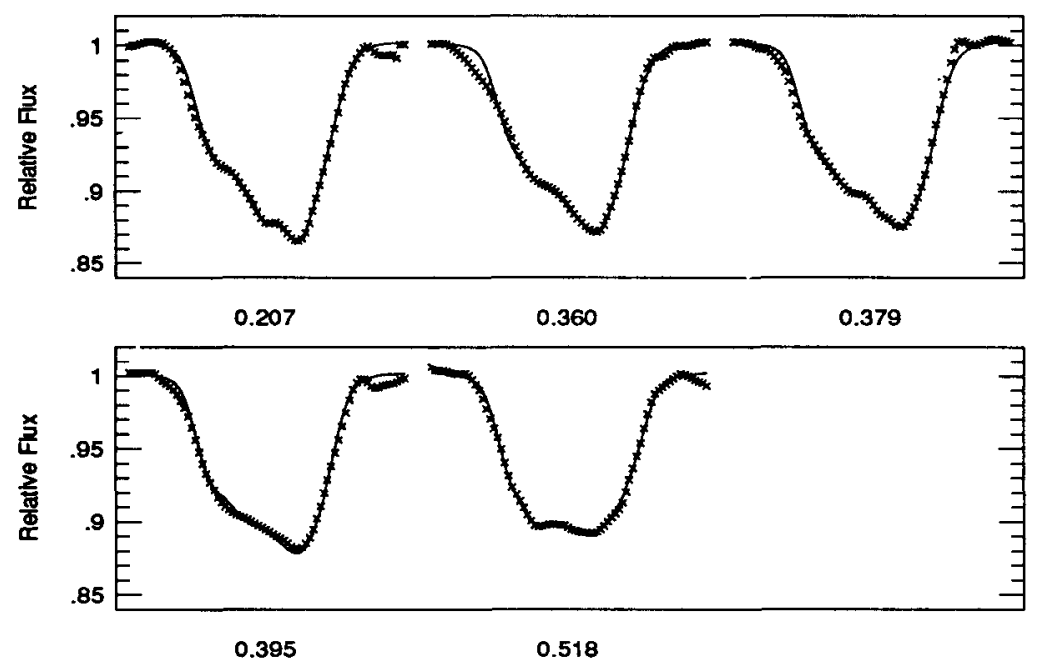

Fig. 3. The observed spectral line profiles (crosses) and linefits for the November data set.
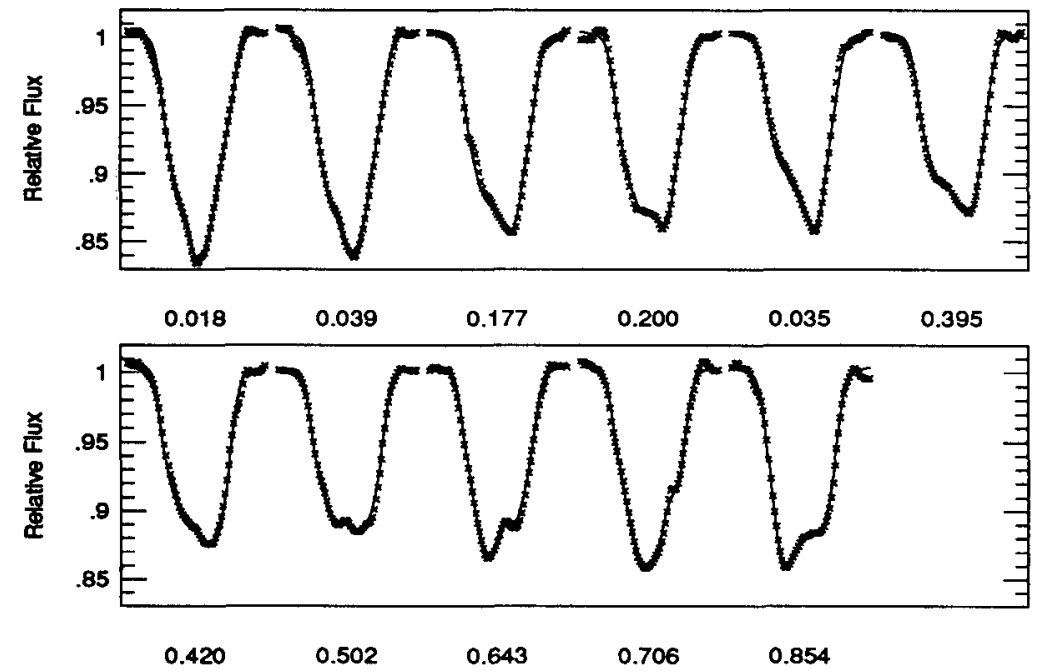

Fig. 4. The observed spectral line profiles (crosses) and linefits for the January data set.

were used to take into account the center to limb variations in the line profile. The limb darkening coefficient in Table 1 represents an approximation to the limb darkening coefficient produced by the model atmospheres. The spectral line profile for the spot was taken to be a scaled version of the photospheric profile. This assumes, naturally, that the line in the spot has the same shape as in the photosphere and thus does not change its equivalent width with temperature. This is not strictly 
true as the $\mathrm{Ca}$ I line increases in strength with decreasing temperature. We feel, however, that this is a valid representation for the spot profiles for two reasons. First, extensive testing in Paper I indicated that the final Doppler image is insensitive to changes in the line strength in the spot. This is primarily due to the fact that the spots are dark and do not contribute a significant fraction of the flux to the integrated profiles. The changes in the integrated line flux shapes therefore come primarily from changes in the continuum level and not from changes in line strength. Secondly, in our observations of the RS CVn's we find that the shape of the Fe I $6430 \AA$ line, which has a weaker temperature dependence than the Ca I line, is always a scaled version of the $\mathrm{Ca}$ I line. This would not be the case if the spot contribution to the spectral line profiles were significant.

Table 1: Stellar Parameters

\begin{tabular}{|ccc|}
\hline Parameter & Primary & Secondary \\
\hline Spectral Type & K0IV & G5V \\
$v$ sin $i$ & $39 \pm 1 \mathrm{~km} \mathrm{~s}^{-1}$ & $7.25 \pm 0.25 \mathrm{~km} \mathrm{~s}^{-1}$ \\
$i$ & $60^{\circ}$ & - \\
$\mathrm{T}_{\text {phot }}$ & $4750 \mathrm{~K}$ & $5700 \mathrm{~K}$ \\
$\log \mathrm{g}$ & 3.5 & 4.27 \\
$\xi_{r t}$ & $5 \mathrm{~km} \mathrm{~s}^{-1}$ & $3.5 \mathrm{~km} \mathrm{~s}^{-1}$ \\
$\epsilon$ & 0.64 & 0.6 \\
\hline
\end{tabular}

\subsection{The Doppler images}

The Doppler images derived from the three data sets are shown in Figures 5-7. The reconstruction procedure uses the principles of maximum entropy reconstruction as outlined in Paper I. Each figure shows the star in a "mashed" polar projection. Latitude lines are drawn equally spaced at intervals of $4.5^{\circ}$ down to a latitude of $-50^{\circ}$. Each square represents an image pixel of $4.5^{\circ} \times 4.5^{\circ}$. A total of 2310 pixels was used in the modeling. The darkest latitude circle represents the stellar equator while medium dark latitude lines are drawn every $30^{\circ}$. The star rotates in a counter-clockwise manner for an observer looking up from phase 0.0 .

The radial tic marks surrounding the star represent those phases at which observations were made. In Figures 5 and 6 , the missing pixels represent phase 'gaps' of missing pixel information. At a given phase, pixels about \pm 0.2 in phase from the center make a discernible contribution to the observed line profile. Naturally, those pixels near the limb make less of a contribution due to foreshortening and ideally one should display the pixels in a manner that is weighted to their visibility. At the present time there is no convenient way for us to do this. Instead we chose only to display those pixels for which we have at least some information.

Pixels contained in the spotted region are marked with an ' $\mathrm{X}$ ' and thus the displayed images represent a binary brightness distribution (spot or no spot). The actual Doppler images produce a smooth variation in brightness between spot and photosphere. Also the maximum entropy method tends to favor producing a 


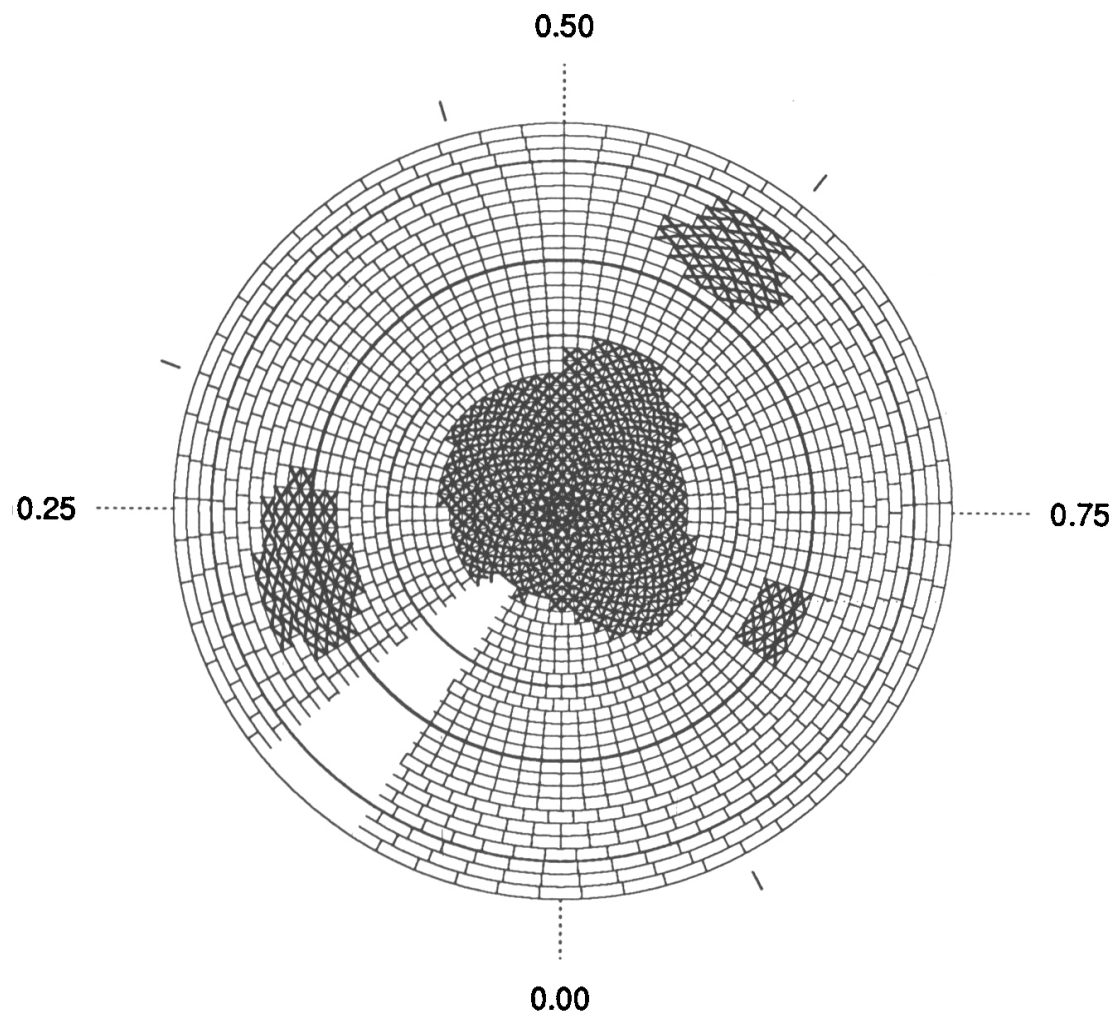

Fig. 5. Doppler Image of UX Arietis in August 1986.

large warm feature as opposed to a small cool one. We therefore must decide, in a consistent manner, below what intensity level a pixel must have in order to be in a 'spot'. This thresholding procedure was chosen as follows. A temperature of 3550 $\mathrm{K}\left(\Delta \mathrm{T}=1200^{\circ}\right)$ was chosen for the spot, slightly warmer than the value of 3360 $\mathrm{K}$ found by Poe and Eaton (1985) using photometry. A threshold value is chosen such that all pixel values below this threshold are replaced by the spot temperature and all pixels above the threshold are replaced by the photospheric temperature. The integrated line profiles from this thresholded image are then compared to the observed profiles. The threshold level is then varied until a good match is made between the observed and computed profiles. This procedure essentially turns the original continuous brightness distribution into a discrete 2-level one that is still consistent with the observed profiles. Experience has also shown that for those Doppler images for which published photometry is available, images thresholded in this way are also consistent with the photometry.

Figure 5 shows the thresholded Doppler image for UX Arietis in August 1986. The spot distribution is characterized by a large polar spot and three low-latitude spots; one near the equator at phase 0.25 , one at latitude $-25^{\circ}$ and phase 0.59 and 


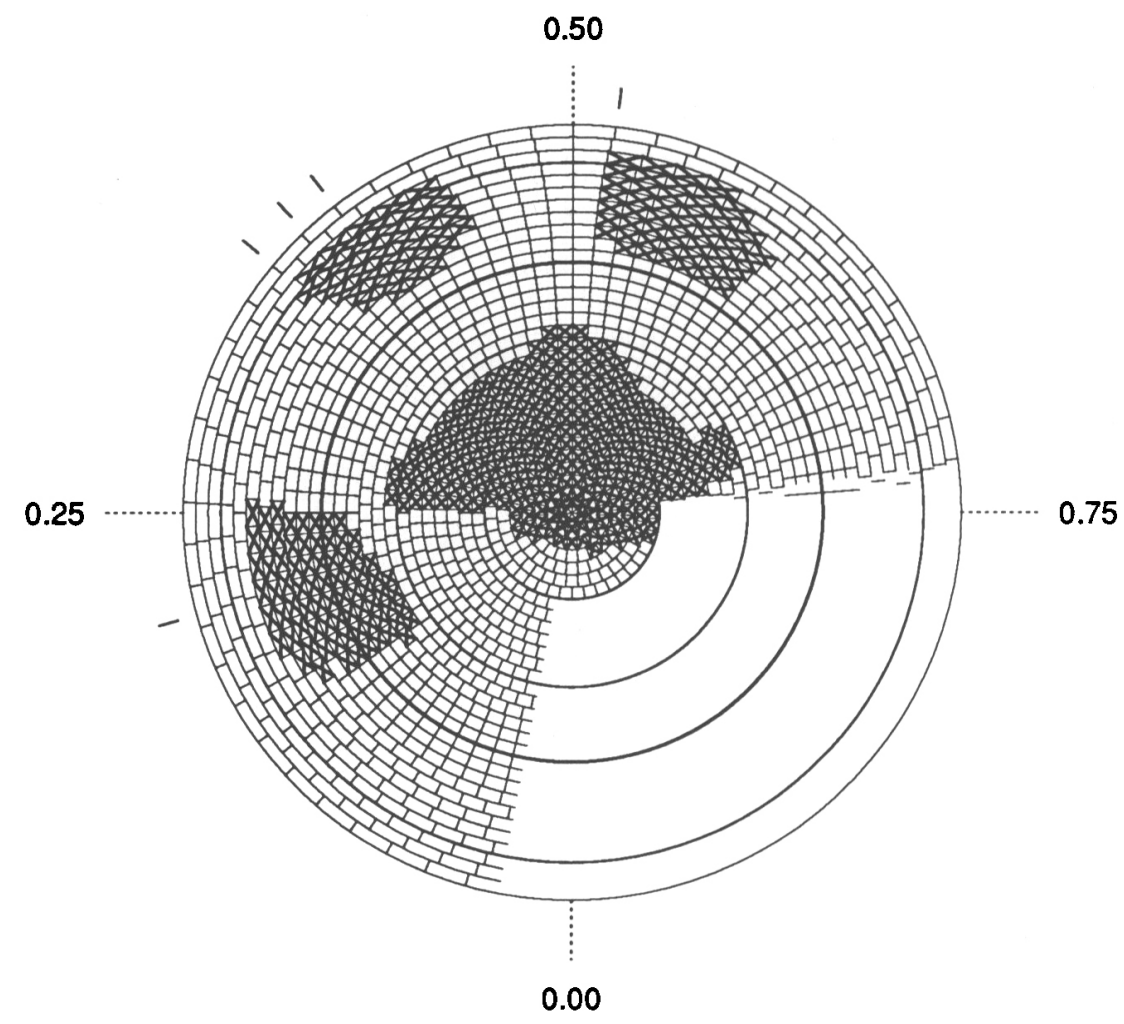

Fig. 6. Doppler Image of UX Arietis in November 1986.

another at $+10^{\circ}$ and phase 0.85 . Note that the polar spot has two appendages: one at phase 0.56 and another near phase 0.88 .

The spot distribution for UX Arietis derived from the November data set is shown in Figure 6. Both low-latitude spots from the August image are still present although the one below the equator near phase 0.6 has migrated counter-clockwise (to a lower phase). Note that a new low-latitude spot near phase 0.40 has also appeared. The reality of this spot is somewhat uncertain since it is at such a low latitude and it may be a 'shadow' of the polar spot due to poor phase coverage. There are also several polar appendages two of which (at phase 0.5 and 0.69 ) appear to have counterparts in the August image.

The Doppler image of UX Arietis in January 1987 is shown in Figure 7. Two of the low-latitude features from the November image are present in the January image. However, the low-latitude spot which was present at phase 0.40 in the November image (but not in the August image) has disappeared. This leads us to believe that this spot in the November image was real and not a shadow of the polar spot because the phase coverage for this part of the star was the same as for the November image and no such shadow spot appears in the January image. The ultimate fate of this spot is unknown at the present time due to 


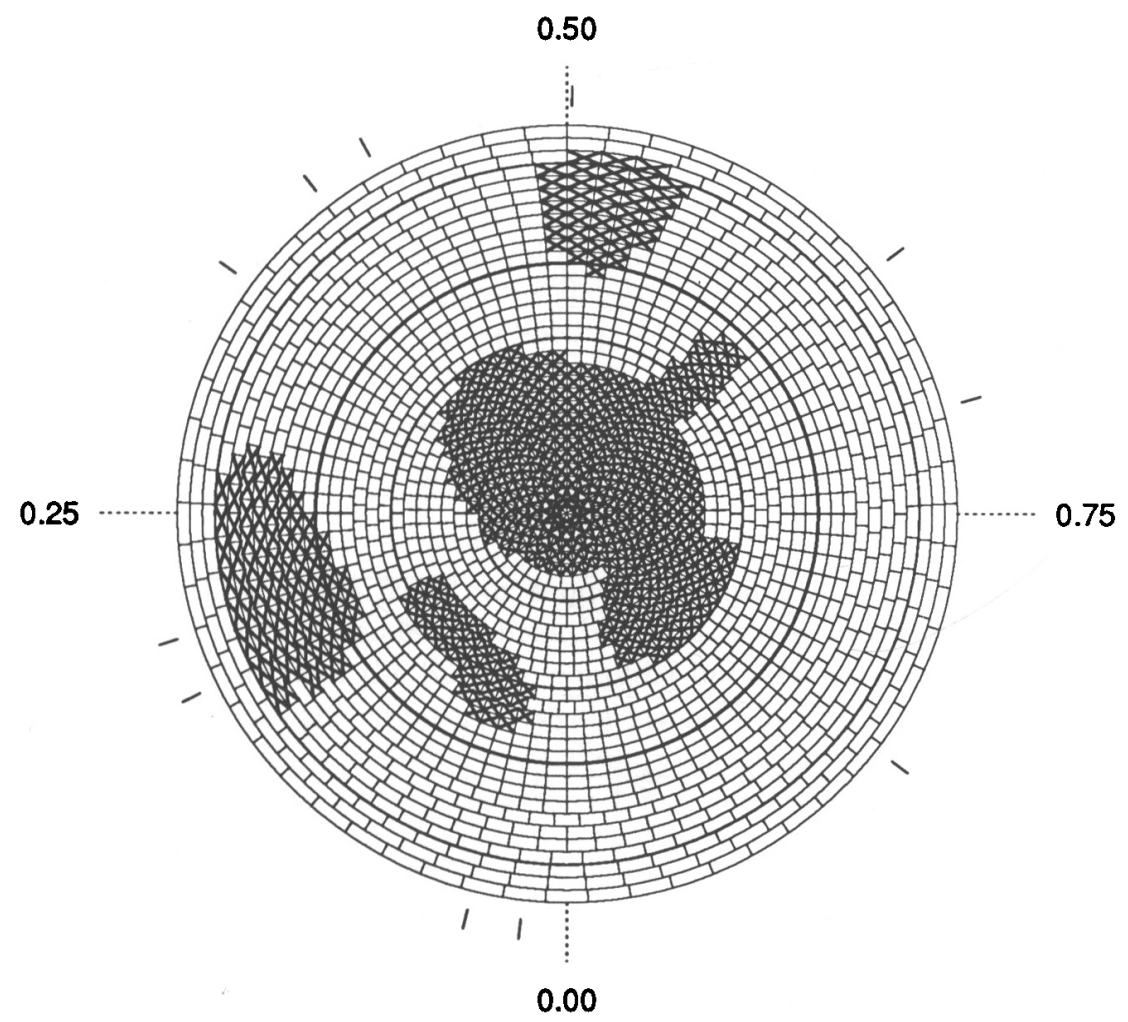

Fig. 7. Doppler Image of UX Arietis in January 1987.

the poor time resolution of the Doppler image sequence. This spot could have simply disappeared (possibly by submerging back into the subphotospheric layers) or it could have migrated in latitude and/or longitude from its original position in the November image. Most likely the spot migrated towards the south pole since a small amount of southern motion would cause the spot to drift out of view. Significant longitudinal drift seems unlikely due to the apparent absence of phase smearing for this feature in the November image as well as the fact that its disappearance in the January image would require a merger with one of the other low-latitude spots. This requires a much larger migration rate than is observed for the other features. The January image also marks the appearance of a mid-latitude spot near phase 0.1 as well as the disappearance of the $+10^{\circ}$ latitude spot from the August image. The lack of phase coverage near this latter spot in the November image prevents us from knowing the fate of this spot although we strongly suspect that it migrated polewards possibly becoming part of the polar appendage near phase 0.85 in the January image. 


\section{Differential rotation in UX Arietis}

Although the spot distribution on UX Arietis visibly changes in the sequence of images presented in Figures 5-7, there are certain features which one can clearly follow from image to image. These include two polar appendages (at phases 0.43 and 0.63 in the January image), and two low latitude spots (near phases 0.2 and 0.6 in the August image). This enables us to measure the differential rotation rate for UX Arietis over a latitude range of $-29^{\circ}$ to $+45^{\circ}$.

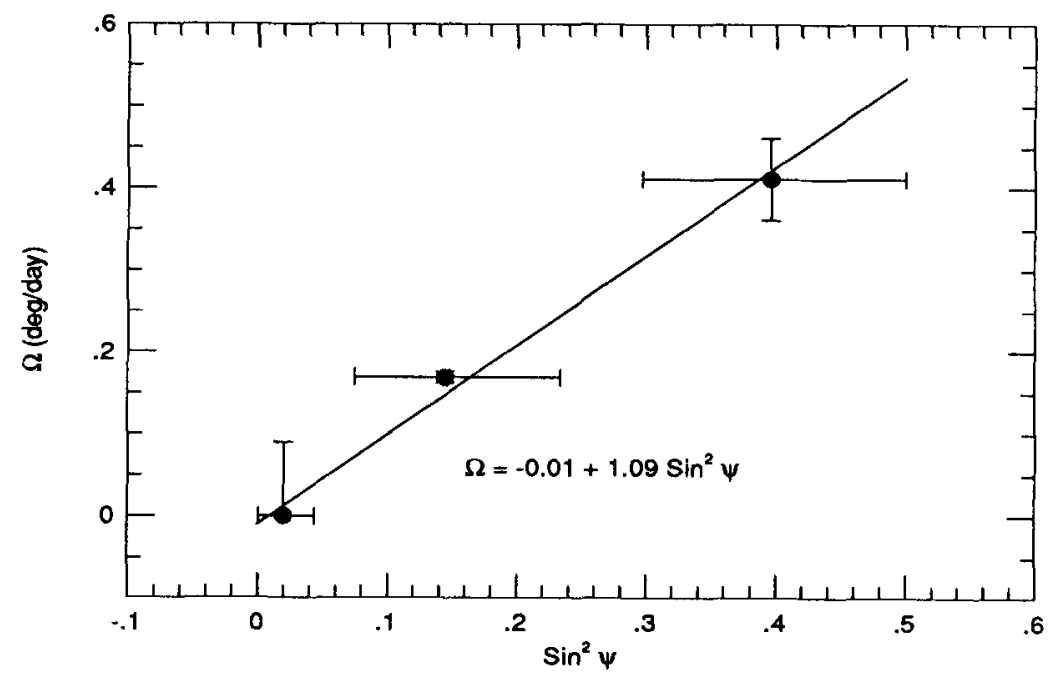

Fig. 8. The rotation rate (relative to the orbit) of UX Arietis as a function of the sinesquared of the latitude, $\phi$.

The resulting differential rotation measured with respect to the reference frame of the orbit is shown in Figure 8. The least squares fit to the data yields:

$$
\Omega_{\text {rel }}=-0.01+1.09( \pm 0.09) \sin ^{2} \phi(\% \text { day })
$$

The first term on the right hand side of Eqn. 1 represents the rotation rate of the equator with respect to the orbital frame. To within our uncertainties, it is zero, indicating that the equator is tightly synchronized to the orbital angular velocity. The second term gives the degree of latitudinal shear and, being positive, indicates that the angular velocity increases with increasing latitude (polar acceleration). This measured differential rotation as measured from various features in the images was consistent in that the rates derived from the polar and low-latitude features between the August and November images were consistent with those derived from the November and January images.

By comparison the solar value (relative to the solar equator) is:

$$
\Omega_{r e l}=-3.0 \sin ^{2} \phi(\% / d a y)
$$


where $\phi$ is the stellar latitude.

The amount of differential rotation may be parameterized by $\alpha$, the ratio of the difference between polar and equatorial velocities to the equatorial velocity:

$$
\alpha=\frac{\Omega_{\text {equator }}-\Omega_{\text {pole }}}{\Omega_{\text {equator }}}
$$

Solid body rotation results in $\alpha=0$, whereas equatorial acceleration has $\alpha>0$ and polar acceleration results in $\alpha<0$. The differential rotation law for UX Arietis is $\alpha=-0.02 \pm 0.002$ whereas $\alpha=+0.197$ for the Sun.

\section{Discussion}

The most surprising result from our sequence of images is that the differential rotation in UX Arietis is small and negative, that is, the poles rotate faster than the equator. In the case of the Sun the coupling between rotation and convection may be able to explain the equatorial acceleration (Glatzmaier, 1988; Gilman and Miller, 1986), however, one must be very wary of applying the solar analogy to the RS CVn stars. For one, the structure of UX Arietis is quite different from that found in the Sun; UX Arietis is more evolved and has a much larger radius and rotation rate than the Sun. It also has a much deeper convection zone. Also, UX Arietis is in a close binary system and tidal forces certainly must play a role in controlling stellar motions, unlike for the solar case. Clearly UX Arietis may play a key role for testing theories of dynamos and differential rotation.

The synchronization of the equator of UX Arietis with the orbital period is no coincidence since UX Arietis nearly fills its Roche lobe (Huenemoerder et. al., 1989) and one expects tides to play a dominant role in determining the rotation rate. Also $88 \%$ of all $\mathrm{RS}$ CVn systems exhibit synchronization with the orbital period. What is not known is what role tidal stresses have in suppressing (or creating) differential rotation in UX Arietis. One may naively think that tidal stresses would work to keep the rotation rate of the various latitude bands the same. However, theoretical calculations by Scharlemann (1982) suggest that tides in RS CVn binary systems should not suppress differential rotation.

Scharlemann (1982) also predicted that, when the tidal coupling torque vanishes (maximum degree of synchronism achieved), part of the star should be rotating faster than the orbit, and part more slowly. Co-rotation with the orbit should occur at some intermediate latitude (the co-rotation latitude). This concept has often been proposed to explain the observed forward and reverse migration of waves in the RS CVn photometric curves as spots migrate above or below the co-rotation latitude. However, if our differential rotation results are correct, UX Arietis does not fit this model. Rather, the co-rotation latitude is at the equator, and no part of the star rotates more slowly than the orbital velocity. Perhaps the tidal coupling torque has not yet vanished and UX Arietis is not yet in a state of maximum synchronization. 
Furthermore, if, as we believe, no part of the star rotates more slowly than the orbit, then one should never see migration of the photometric 'wave' to later phases in the light curves. Rather, the wave should always migrate to earlier orbital phases. Busso et al. (1986) do find a dominant trend for migration to earlier phases as expected from our result, but also find times where the wave migrates to later phases. We suspect that the latter must be attributable to changes in the brightness centroid of a complex spot distribution rather than to drift of the longitude of some presumed single 'effective' spot. Such observations of migration of the wave to later phases on UX Arietis may indicate that wave migration is not a reliable indicator of differential rotation.

In spite of the very small amount of apparent differential rotation at the surface, UX Arietis shows a great deal of activity indicative of the presence of a very powerful dynamo. If differential rotation is indeed a necessary ingredient for dynamo action, then it must either be hidden below the surface of UX Arietis, or else the spot motions are not reflecting the true surface latitudinal shear (i.e. the spots are anchored to a deep and more uniformly rotating inner zone).

Finally, it is clear that the spot distribution on UX Arietis can change on time scales of a month. This is evident from changes in the shape of the polar spot as well as the appearance and disappearance of isolated spots. This demonstrates that one must exercise great care in using photometric light curves for deriving differential rotation rates. This also shows that to accurately derive the morphology of spots on the surface of these stars, one would like at least 3 or more Doppler images a year. This requires prodigious amounts of observing time and severely taxes the resources of most major observatories. Collaborative observing efforts between several observatories is required in order to get adequate time resolution between images as well as to get complete phase coverage.

\section{References}

Bell, R.A., Eriksson, K., Gustafsson, B., Norlund, $\AA .:$ 1976, Astron. Astrophys. Suppl. 23, 37

Busso, M., Scaltriti, F., Cellino, A.: 1986, Astron. Astrophys. 156, 106

Gilman, P., Miller, J.: 1986, Astron. Astrophys. Suppl. 61, 585

Glatzmaier, G.: 1985, Astrophys. J. 291, 300

Huenemoerder, D., Buzasi, D., Ramsey, L.: 1989, Astron. J., 98,1398

Poe, C., Eaton, J.: 1985, Astrophys. J. 289, 644

Scharlemann, E.: 1982, Astrophys. J. 253, 298

Vogt, S.S., Penrod, G.D., Hatzes, A.P.: 1987, Astrophys. J. 321, 496 